\title{
Absolute intensities of the $\gamma$-ray emissions originating from the electron capture decay of ${ }^{153} \mathrm{Gd}$
}

\author{
R. Shearman ${ }^{1,2, a}$, S.M. Collins ${ }^{1}$, J.D. Keightley ${ }^{1}$, A.K. Pearce ${ }^{1}$, and J. Garnier ${ }^{1,3}$ \\ 1 National Physical Laboratory, Hampton Road, Teddington, Middlesex TW11 0LW, UK \\ 2 Department of Physics, University of Surrey, Guildford GU2 7XH, UK \\ ${ }^{3}$ Grenoble Institute of Technology, 38016 Grenoble Cedex 1, France
}

\begin{abstract}
Gd}$ has widespread use, in non-destructive testing, as a line source in SPECT imaging and has been recently proposed as an in-vitro interstitial rotating shield brachytherapy (I-RSBT) source. In this work, the six most intense emissions in the de-excitation of the daughter nucleus ${ }^{153} \mathrm{Eu}$ have been measured, with an improved accuracy and precision to $\gamma$-ray emission intensities reported previously, via two characterised HPGe spectrometers. A specific absolute activity of 512.5 (25) $\mathrm{kBq} \mathrm{g}^{-1}$ was determined using the $4 \pi$ (LS)- $\gamma$ digital coincidence counting technique. This absolute activity was used to determine an absolute intensity for the $97.4 \mathrm{keV} \gamma$-ray emission of 30.15 (20) per 100 decays. The reported absolute emission intensity of this transition in this work has a relative difference of $4 \%$ from the currently recommended value.
\end{abstract}

\section{Introduction}

Gadolinium-153 is used in nuclear medicine as part of a line tracer in SPECT imaging [1-3], where attenuation corrections must be known on a patient-by-patient basis. Historically it has been used in bone absorptiometry [4-6], to study bone mineral density and to help diagnosis of osteoporosis. Recently, this radionuclide has been used for non-destructive testing of metal pipes [7] due to the strong dual emissions of the $97.4 \mathrm{keV}$ gamma-ray emission and the K X-rays. Gadolinium-153 has been proposed as a replacement for ${ }^{192} \mathrm{Ir}$ in brachytherapy treatment. The lower energy photon emissions from ${ }^{153} \mathrm{Gd}$ are advantageous as they would require less shielding $[8,9]$. The common production mode of ${ }^{153} \mathrm{Gd}$ is by the neutron irradiation of a europium target, which is subsequently chemically separated to produce a radiochemically pure ${ }^{153} \mathrm{Gd}[10,11]$.

The ground state of ${ }^{153} \mathrm{Gd}$ decays by electron capture (EC) $(100 \%)$ to ${ }^{153} \mathrm{Eu}$. The decay scheme and relative intensities evaluated by the Decay Data Evaluation Project (DDEP) is shown in Fig. 1. Two evaluations of the decay scheme of ${ }^{153} \mathrm{Gd}$ have been proposed in the new millennium $[12,13]$, these show a discrepant number of excited levels being populated in ${ }^{153} \mathrm{Eu}$, and both discuss the need for new measurement due to strong disagreement in $P_{\gamma}$ values for weakly populated gamma-ray emissions in the daughter nuclei.

A BIPM [14] report detailing the methodology of the SIRIC [15] computer model for the ${ }^{226} \mathrm{Ra}$ equivalent activity measurements of the BIPM ionisation chambers comprising the "International Reference System", identified ${ }^{153} \mathrm{Gd}$ as a possible radionuclide with inconsistent decay data. The modelled data was an outlier with a relative difference of $4.3 \%$. Such a sizeable difference

a e-mail: Robert.shearman@npl.co.uk was not observed in radionuclides of similar photon decay profiles.

These observations are a cause of concern for a radionuclide proposed for in-vitro cancer treatment. It is therefore important to measure the intensity of the internal transitions within ${ }^{153} \mathrm{Eu}$ following the EC decay of ${ }^{153} \mathrm{Gd}$.

In this work the absolute activity of a solution of ${ }^{153} \mathrm{Gd}$ has been determined by $4 \pi$ (Liquid scintillation (LS)) $-\gamma$ Digital Coincidence Counting (DCC) [16], which has subsequently been used to determine the ${ }^{153} \mathrm{Gd}$ absolute $\gamma$-ray emission intensities by high purity $\gamma$-ray spectrometry. Throughout this article, uncertainties are stated as standard uncertainties or combined standard uncertainties as defined in the Guide to the Expression of Uncertainty in Measurement (GUM) [17].

\section{Experimental conditions}

\subsection{Sample preparation}

An aqueous solution of radiochemically pure ${ }^{153} \mathrm{GdCl}_{3}$ in $0.5 \mathrm{M} \mathrm{HCl}$ with $50 \mu \mathrm{g} \mathrm{g}^{-1}$ stable $\mathrm{Gd}$ carrier was used in this work. The sources for the $4 \pi(\mathrm{LS})-\gamma$ DCC technique were prepared by gravimetrically dispensing one drop of this solution $(0.1 \mathrm{mg}-0.3 \mathrm{mg})$ to twelve $20 \mathrm{~mL}$ glass liquid scintillation vials containing $0.1 \mathrm{~mL}$ of carrier and $10 \mathrm{~mL}$ of Ultima Gold AB scintillant. $1 \mathrm{~g}$ aliquots of the solution were gravimetrically dispensed into three British Standard (BS) [18] $2 \mathrm{~mL}$ flame sealed ampoules for measurement by gamma-ray spectrometry.

\subsection{HPGe gamma-ray spectrometry}

\subsubsection{Gamma-ray spectrometers}

Two ORTEC n-type High Purity Germanium (HPGe) $\gamma$-ray spectrometers were used to determine the absolute emission intensities of ${ }^{153} \mathrm{Gd}$. The detectors were identified

(C) The Authors, published by EDP Sciences. This is an Open Access article distributed under the terms of the Creative Commons Attribution License 4.0 (http://creativecommons.org/licenses/by/4.0/). 


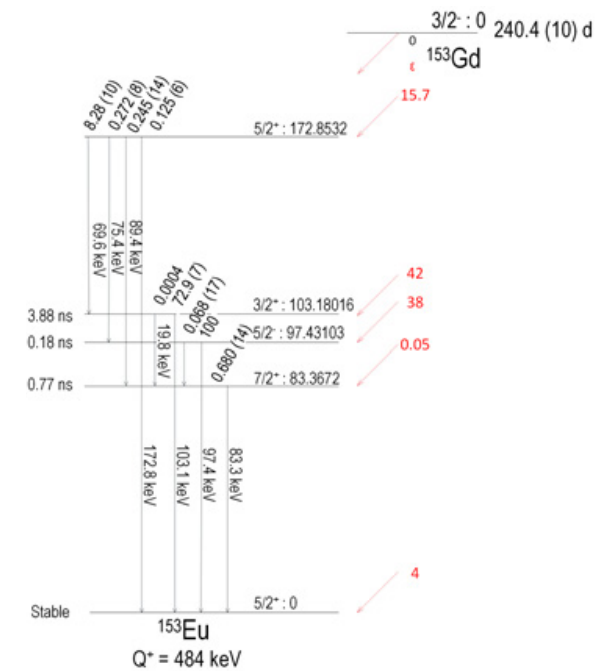

Figure 1. The decay scheme of ${ }^{153} \mathrm{Gd}$ as reported by Bé [12]. Several levels proposed to be populated [13] during this decay; the first $7 / 2^{-}$and the tentative second $7 / 2^{+}$state are omitted.

as 'BART' and 'LOKI'. The respective relative efficiency of 'BART' and 'LOKI' are $28 \%$ and $22 \%$, with a resolution (FWHM) of $1.78 \mathrm{keV}$ and $1.68 \mathrm{keV}$ at $1332 \mathrm{keV}$. In addition, LOKI has a resolution of $676 \mathrm{eV}$ at $122 \mathrm{keV}$.

Both detectors were contained in a $1.5 \mathrm{~m} \times 1 \mathrm{~m} \times 1 \mathrm{~m}$ lead shield, with walls $10 \mathrm{~cm}$ in depth to reduce the effects of external background radiation. To reduce the interference of the $\mathrm{Pb}$ fluorescence $\mathrm{X}$-rays, degraders of $0.5 \mathrm{~mm} \mathrm{Cd}$ and $0.7 \mathrm{~mm} \mathrm{Cu}$ line the inside of the shield. The shields have aluminium optical bread boards mounted along the horizontal axis of the shield, which were used to mount kinematic mounting plates to which precision engineered sample holders could be attached. This enabled highly reproducible geometric sample positioning in front of the spectrometer (reproducibility uncertainty of $0.016 \%$ ). The full-energy peak (FEP) detection efficiency of both spectrometers were determined for the matching geometry (to the samples) using a suite of sources traceable to primary standards of radioactivity. The sample-to-endcap distance and solid angle $(\Omega)$ for BART and LOKI were approximately $250 \mathrm{~mm}(\Omega \sim 0.038 \mathrm{sr})$ and $290 \mathrm{~mm}(\Omega \sim 0.045 \mathrm{sr})$ respectively. The distance between source and detector was used as to reduce the effects of dead-time and pulse pile-up. A further benefit to this extended sample-to-detector geometry is the reduction of True Coincidence Summing (TCS) to negligible levels due to the low $\Omega$ (relative to the standard uncertainties of the measurements). The FEP detection efficiency curve for both detectors were fitted using the procedure previously detailed in Collins et al. [19], which takes into consideration the correlations in standard uncertainties of the calibration points arising from, for example, the nuclear data, activity standardisation. The FEP detection efficiency and corresponding residuals for 'LOKI' are shown in Fig. 2. The FEP detection efficiency for the HPGe spectrometer 'BART' has been previously described by Collins et al. [19].

Spectra were acquired using a chain of analogue electronics (CANBERRA AFT Research amplifier 2025, Analogue-to-Digital Convertor 8715, AIM) connected to a computer running CANBERRA GENIE 2000 v2.1c
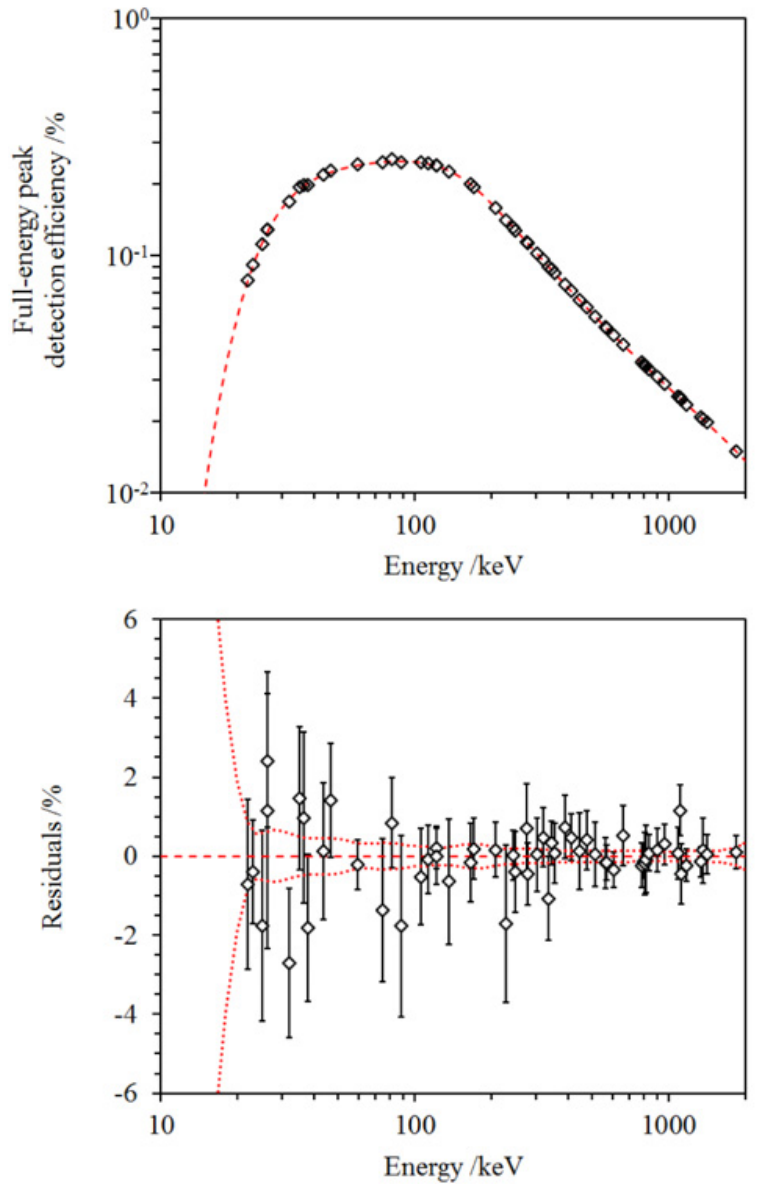

Figure 2. The full-energy peak detection efficiency curve of the HPGe gamma-ray spectrometer 'LOKI', covering the energy region from approximately $20 \mathrm{keV}$ to $1840 \mathrm{keV}$. The residuals of the quartic b-spline fit to the measured data is shown in $b$ ). The uncertainty bars in b) represent the standard uncertainty of each data point and the dashed line represents the standard uncertainty of the FEP detection efficiency curve over the calibrated energy range.

software. The net peak area losses due to dead time and pulse pile-up were corrected using separate methods for each detector. For BART, the losses were corrected using the integrated pile-up rejection/live-time correction circuit with an additional empirically determined correction factor for those events not captured by the integrated circuit. LOKI used the well-established reference pulse method to correct the peak areas for both effects.

The neutron irradiation of a natural Europium target produces more than the desired radioactive isotope [10], it is therefore possible that the source could be contaminated with several other isotopes. In this study over 5 years, no contaminant peaks could be identified, except for emissions attributed to NORM, lead X-rays, or sum peaks. The first spectrum was acquired 7 days after preparation and was consistent with results taken much later in the study ( $\sim 5$ years), this increases confidence that the solution was clean of contamination.

Fourteen measurements were made of the three prepared ampoules. Acquisitions lasted between $86400 \mathrm{~s}$ and $250000 \mathrm{~s}$. The integrated count rates and dead time were of the order $1600 \mathrm{cps}$ and $6.7 \%$ respectively for the initial measurements. A measured spectrum of ${ }^{153} \mathrm{Gd}$ can be seen in Fig. 3. 


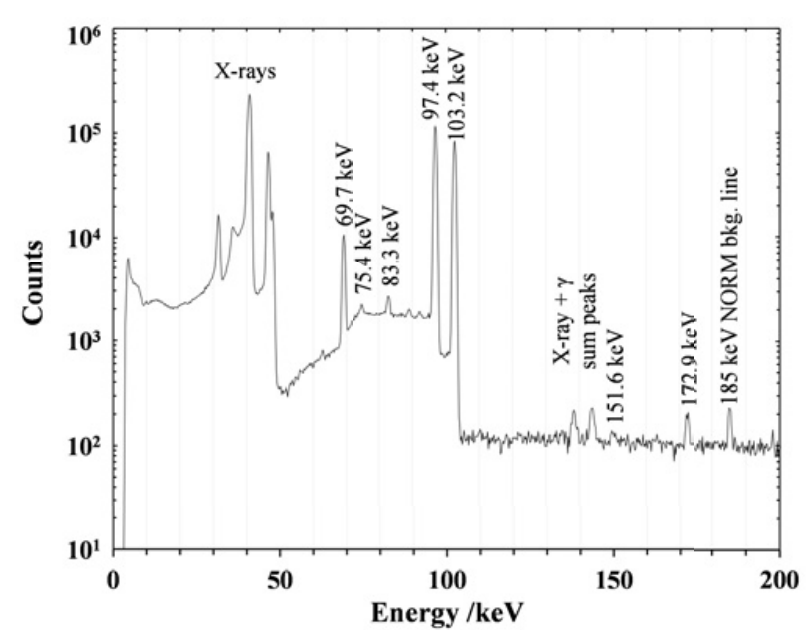

Figure 3. An annotated spectrum of ${ }^{153} \mathrm{Gd}$ measured by the HPGe $\gamma$-ray spectrometer 'LOKI'.

The spectra were analysed using the GENIE 2000 software, with the fit to each peak manually reviewed and modified where necessary using the GENIE Interactive Peak Fit application. The full-energy peak areas were corrected for background, decay during the measurement period, pulse pile-up and self-absorption.

\subsection{Digital coincidence counting (DCC)}

The $4 \pi(\mathrm{LS})-\gamma$ coincidence system at the National Physical Laboratory (NPL) consists of a LS counter populated with two Hamamatsu R331-08 head-on type Photomultiplier Tubes (PMTs) operated in coincidence to provide a significant reduction in background count rates, due to the elimination of sporadic thermionic emissions from the photocathodes. The $\gamma$-channel utilised a $76 \mathrm{~mm} \times 76 \mathrm{~mm}$ $\mathrm{NaI}(\mathrm{Tl})$ scintillation crystal, situated below the LS light guide in a lead shield.

The amplified pulses resulting from both detector systems were digitised and processed by the NPL Digital Coincidence Counting system, which has been described elsewhere [16,21,22]. This digital system handles the offline imposition of dead-times in both channels, selection of photon windows, imposition of appropriate delay/s and coincidence resolving times and provides data in a format suitable for the "Computer Discrimination" efficiency extrapolation method described by Smith [23,24].

Absolute activity determinations of $\mathrm{EC}$ radionuclides using coincidence techniques are complicated due to the low detection efficiency of the counter for X-rays and Auger electrons $(\mathrm{X}, \mathrm{e})$ from L, M and higher-shell capture events and following L, M conversions. The elevated non-detection of the initial capture $(\mathrm{X}, \mathrm{e})$ events leaves the counter open to register correlated events such as $\gamma$-rays and conversion electrons, requiring appropriate corrections. The usual technique of performing efficiency extrapolation to avoid calculation of these corrections, requires measurement of the count rates $\mathrm{N}_{L S}, \mathrm{~N}_{\gamma}, \mathrm{N}_{c}$ in the $4 \pi \beta 4 \pi(\mathrm{LS})$ channel, the gamma channel and the coincidence channel respectively, over a range of values of the LS-counter efficiency (estimated as $\mathrm{Nc} / \mathrm{N} \gamma$ ), facilitating extrapolation to unit efficiency and yielding an estimate of the source activity.

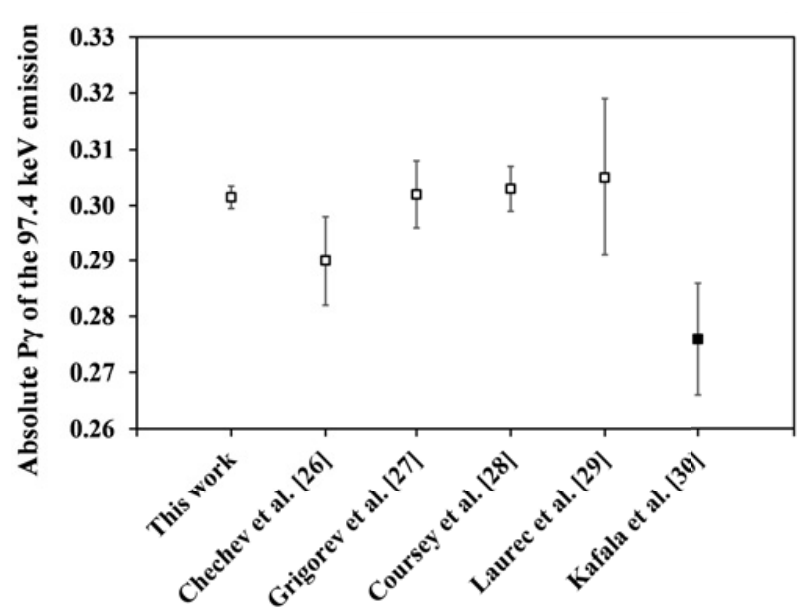

Figure 4. The evaluated value of the absolute intensity of the $97.4 \mathrm{keV}$ emission, the value reported by Chechev, is below that of this work and of Grigorev. Coursey and Grigorev are measured values [29-31]. Solid data points represent calculated data.

However, for EC nuclides, the magnitude of the effects of loss of $(\mathrm{X}, \mathrm{e})$ events is dependent on the ratios of probabilities for $\mathrm{K}$ and L-capture between the various branches, as coherently described by Funck and Nylandstedt-Larsen [25], and may produce a residual bias in the efficiency extrapolation result, even after extrapolation. To minimise this bias, a bi-dimensional efficiency extrapolation [26] was performed with the first gate set on the K X-rays between $41 \mathrm{keV}$ and $49 \mathrm{keV}$ (efficiency typically varied from $84 \%$ to $77 \%$ ), and the second gate encompassing the $97 \mathrm{keV}$ and $103 \mathrm{keV}$ gamma rays (efficiency typically varied from $75 \%$ to $63 \%$ ). When simultaneously extrapolating both channels to unit efficiency, the resulting estimate of the activity per unit mass was $512.2(25) \mathrm{kBqg}^{-1}$. Gating on the $\mathrm{K}$ X-rays, $97 \mathrm{keV}$ and $103 \mathrm{keV} \gamma$ rays individually yielded biases of $0.13 \%$ and $0.3 \%$ respectively, with significantly larger variances.

\section{Results and discussion}

\subsection{Relative $\mathbf{P}_{\gamma}$ measurements}

In total, there are 14 prompt gamma-ray transitions observed in the decay of ${ }^{153} \mathrm{Gd}$ [12]. In this work, six of the $\gamma$-ray emissions have been identified and reported. The low-energy emissions $(<25 \mathrm{keV})$ of this decay scheme have not been observed due a combination of factors; low intensities, interference from X-rays and Compton scattering, reduced efficiency. The $54.1 \mathrm{keV}$ has not been observed in any measurements. The possible $166.1 \mathrm{keV}$ transition, which would connect the second $7 / 2^{+}$level that is proposed to be populated by the decay of ${ }^{153} \mathrm{Gd}$, is measured with inconsistency in these data, this is also true of the $68.2 \mathrm{keV}$ transition linking the $7 / 2^{-}$ $151.6 \mathrm{keV}$ and the first $7 / 2^{+} 83.3 \mathrm{keV}$ levels in ${ }^{153} \mathrm{Eu}$. The stronger populated $75.4 \mathrm{keV}$ and $83.3 \mathrm{keV}$ transitions are not reported, although identified in all spectra, due to inconsistent results. This inconsistency is due to the complexities of the Compton continuum in these energy regions.

The absolute emission intensity determined for the $97.4 \mathrm{keV} \gamma$-ray, using the absolute activity determined by 
Table 1. The reported values of this work compared to the evaluated data. This work consistently is in disagreement with the most recent DDEP evaluation [12]. Absolute data is calculated using the DCC calculated value for the absolute intensity of the $97.4 \mathrm{keV}$ transition.

\begin{tabular}{|c|c|c|c|}
\hline Energy & \multicolumn{2}{|c|}{ This work } & DDEP [12] \\
\hline$/ \mathrm{keV}$ & $\mathrm{I}_{\gamma}$ & $\mathrm{I}_{\gamma}(\mathrm{E}) / \mathrm{I}_{\gamma}(97.4)$ & $\mathrm{I}_{\gamma}(\mathrm{E}) / \mathrm{I}_{\gamma}(97.4)$ \\
\hline 69.6 & $2.38(3)$ & $7.89(9)$ & $8.28(10)$ \\
\hline 89.4 & $0.096(5)$ & $0.320(16)$ & $0.245(14)$ \\
\hline 97.4 & $30.15(20)$ & 100 & 100 \\
\hline 103.1 & $22.33(17)$ & $74.06(27)$ & $72.9(7)$ \\
\hline 151.6 & $0.00049(6)$ & $0.0157(10)$ & $0.017(4)$ \\
\hline 172.8 & $0.0396(6)$ & $0.1313(18)$ & $0.125(6)$ \\
\hline
\end{tabular}

Table 2. The uncertainty budget for of the $97.4 \mathrm{keV} \gamma$-ray emission in ${ }^{153} \mathrm{Gd}$.

\begin{tabular}{|l|c|}
\hline Uncertainty component & Value /\% \\
\hline FEP detection efficiency & 0.38 \\
\hline Absolute activity & 0.49 \\
\hline Peak fitting & 0.15 \\
\hline Self-absorption & 0.013 \\
\hline True coincidence summing & 0.10 \\
\hline Gravimetric & 0.02 \\
\hline Geometric reproducibility & 0.016 \\
\hline Dead-time and pulse pile-up & 0.18 \\
\hline Combined uncertainty & $\mathbf{0 . 6 7}$ \\
\hline
\end{tabular}

the DCC technique, was 30.15 (20)\%. The absolute and relative intensities determined in this work are presented in Table 1, the uncertainty budget for the $97.4 \mathrm{keV} \gamma$-ray emission intensity is shown in Table 2.

There is consistent agreement with the relative intensities reported by Chandet al. [27]. The absolute intensity determined for the $97.4 \mathrm{keV} \gamma$ ray differs from the current recommended value of 29.0 (8)\%, which was determined by Chechev [28], with a relative difference of $4.0 \%$. There were clear differences observed between the relative intensities reported by Chechev [28] and this work.

Improved precision in this work with respect to previous measurement is reported for all emissions against all datasets.

The absolute intensities from this work were used to determine a new modelled equivalent activity for the BIPM ionisation chamber using SIRIC [15]. The modelled equivalent activity was found to be 368.4 (19) kBq, the key comparison reference value (KCRV) equivalent activity for this nuclide was 368.1 (17) $\mathrm{kBq}$. This corresponds to a z-score of 0.26 and relative difference of $0.18 \%$, previously the relative difference was $4.3 \%$. The change in relative difference is comparable to the absolute intensity relative difference from this work with the previous evaluated value. This provides evidence that the absolute emission probabilities determined in this work are accurate.

\section{Evaluation of absolute emission intensity of $97.4 \mathrm{keV}$ emission}

A new value of the absolute intensity for this nuclide can be calculated by using a weighted average of the reported values of the absolute intensities which are not reliant on previous $p_{\gamma}$ measurements [28-32]. The work of Laurec [31] differs in that an electron spectrometer was used to measure the internal conversion coefficients, which were then used to calculate the absolute intensities. Other measurements [28-30] used the previously mentioned $4 \pi \beta-\gamma$ liquid scintillation counting used in this work. The equilibrium method used by Laurec is valid only when the decay includes negligible ground state to ground state decays. A $4 \%$ branch in the decay of ${ }^{153} \mathrm{Gd}$ does directly feed the ground state of ${ }^{153} \mathrm{Eu}$.

Several of the values of the absolute intensity for the $97.4 \mathrm{keV}$ reported are not indeed experimentally calculated. Kafala [32] et al, are derived values which we suggest should be omitted from any new evaluation. The current evaluation reports the Geidelman value as described by Chechev [28].

The computed value of the experimental data including the potential outlier reported by Chechev, as identified using Chauvenet's or Pierce's criterion, gives a value of $30.13(17) \%$. This value produces a reduced $\chi^{2}$ of 0.57 far below the critical $\chi^{2}$ of 2.57 for $95 \%$ confidence level. It is suggested that this value should be used in future work needing nuclear decay data for this nuclei.

\section{Conclusion}

Several of gamma emission probabilities for the decay of ${ }^{153} \mathrm{Gd}$ have been measured to a greater accuracy than previously. These results do not agree with literature values. The measured absolute emission intensity of the $97.4 \mathrm{keV}$ gamma ray, 30.15 (20)\% is also in disagreement with the most recent evaluated data. Using this work and previous measurements a simple new evaluation for the absolute intensity results in a value of $30.13(17) \%$, around $4 \%$ greater than the evaluated. This increase in absolute emission probability accounts for the difference seen in the modelled and measured data reported for by the BIPM [14].

The extremely low emission intensity of the $166.2 \mathrm{keV}$ emission, if it indeed is seen in this dataset means that this emission cannot be reported. No evidence is seen that the $269.7 \mathrm{keV} 2^{\text {nd }}$ excited $7 / 2^{+}$level in ${ }^{153} \mathrm{Eu}$ is populated.

Future work on this nuclide is worthwhile, as this evaluation has shown discrepancy. It is believed that a halflife measurement should be considered. This measurement may be of special note as it is the only nuclear data relied upon for the absolute emission probabilities reported in this work.

\section{References}

[1] V. Rodriguez et al., J. Nucl. Med. 57(2), 2833 (2016)

[2] J.A. Case, J. Nucl. Cardiol. 23(5), 1080 (2015)

[3] M.N. Werwick et al., Emission Tomography: The Fundamentals of PET and SPECT (2004)

[4] H.W. Wahner et al., Radiology 156(1), 203 (1985)

[5] A. Moure et al., Phys. Med. Biol. 48(23), 3851 (2003)

[6] A.S.V. Eriksson et al., Acta. Orthop. Scand. 59(1), 19 (1988)

[7] S. Lukose and M. Palanisamy, 3rd. International CANDU In-Service-inspection Workshop \& NDT in Canada 2010 Conference (2010)

[8] S.A. Enger et al, Phys. Med. Biol. 58(4), 957 (2013)

[9] Q.E. Adams, Med. Phys. 41(5), 051703 (2014)

[10] D.W. Ramey, ORNL/TM-10641 (1988)

[11] M.W. Kohring, ORNL/TM-10394 (1987) 
[12] M-M. Bé, et al., Monographie BIMP-5, 2 (2004)

[13] H. Xiaolong, Appl. Radiat. Isot. 68(1), 18 (2010)

[14] M.G. Cox, et al., Monographie BIMP-7 (2007)

[15] C. Michotte, et al., Appl. Radiat. and Isot. 64(10), 1147 (2006)

[16] J.D. Keightley and T.S. Park, Metrologia 44(4), S32 (2007)

[17] BIPM, JCGM 10;2008, Bureau International des Poids et Mesures (2008)

[18] BSI, British Standard BS 795:1983 (1983)

[19] S.M. Collins, et al., Appl. Radiat. Isot. 102, 15 (2015)

[20] M-M. Bé, et al., Monographie BIMP-5, 3 (2004)

[21] J.D. Keightley, Applied modelling and Computations in Nuclear Science ACS Sym, Series 945 (2006)

[22] J.D. Keightley and G.C. Watt, Appl. Radiat. Isot. 56(1), 205 (2002)
[23] D. Smith, Metrologia 11(2), 73 (1975)

[24] D. Smith, Appl. Radiat. Isot. 38(10), 813 (1987)

[25] E. Funck and A. Nylandstedt-Larsen, Int. J. Appl. Radiat. Isot. 34(3), 565 (1983)

[26] D. Smith and L.E.H. Stuart, Metrologia 11(2), 67 (1975)

[27] B. Chand, Appl. Radiat. Isot. 43(8), 997 (1992)

[28] V.P. Chechev and A.G. Egorov, NIM Phys. Res. A. 312(1), 378 (1992)

[29] E.P. Grigorev, et al., Izv.Akad.Nauk SSSR, Ser.Fiz. 45, 795 (1981)

[30] B.M. Coursey, et al., NIM Phys. Res. A. 339(1), 26 (1994)

[31] J. Laurec, et al., NIM Phys. Res. A. 369, 467 (1996)

[32] S.I. Kafala, et al., J. Radioanal. Nucl. Chem. 215(2), 193 (1997) 